same behaviour in the two TLC and GLC systems as testosterone. IR-spectrum of the fermentation compound was idential to that of authentic testosterone acetate.

From these results it can be concluded that progesterone is metabolized by Streptomyces griseus in the same way as by Cladosporium resinae. ${ }^{6}$ This finding supports the hypothesis that this way might be the general route in which the side chain of progesterone is cleaved by microorganisms. ${ }^{6-8}$

The esterase activity in Streptomyces griseus seems to be rather low, even at high $\mathrm{pH}(\mathbf{8 . 3 )}$ at which the esterases generally are stable. The optimum pH for a steroid esterase, prepared from Nocardia restrictus, is $8.0 .^{\circ}$

The rapid disappearance of the progesterone without formation of corresponding amounts of other steroids indicates complete degradation of the steroid moiety to low-molecular compounds. This has been reported for other microorganisms by some authors 10-12 and has also been observed in this laboratory after progesterone fermentations with Penicillium lilacinum and Aspergillus flavus.

1. Vischer, E. and Wettstein, A. Experientia 9 (1953) 371.

2. Peterson, D. H., Eppstein, S. H., Meister, P. D., Murray, H. C., Leigh, H. M., Weintraub, A. and Reineke, L. M. J. Am. Chem. Soc. 75 (1953) 5768.

3. Bodansky, A., Kollonitsch, J. and Wix, G. Experientia 11 (1955) 384.

4. Capek, A., Hanc, O., Macek, K., Tadra, M. and Riedl-Tumova, E. Naturwiss. 43 (1956) 471

5. Fried, J., Thoma, R. W. and Klingsberg, A. J. Am. Chem. Soc. 75 (1953) 5764.

6. Fonken, G. S., Murray, H. C. and Reineke, L. M. J. Am. Chem. Soc. 82 (1960) 5507.

7. Sebek, O. K., Reineke, L. M. and Peterson D. H. J. Bacteriol. 83 (1962) 1327.

8. El-Tayeb, O., Knight, S. G. and Sih, C. J. Biochim. Biophys. Acta 93 (1964) 411.

9. Sih, C. J., Laval, J. and Rahim, M. A. J. Biol. Chem. 238 (1963) 566.

10. Sih, C. J. Biochim. Biophys. Acta 62 (1962) 541.

11. Gibson, P. T., Wang, K. C., Sih, C. J. and Whitlock, H. J. Biol. Chem. 241 (1966) 551.

12. Coombe, R. G., Tsoug, Y. Y., Hamilton, P. B. and Sih, C. J. J. Biol. Chem. 241 (1966) 1587.

Received October 24, 1966.

\section{A Novel Route to 1,3-Dioxoles via a General Acid-Catalyzed Isomerization Reaction}

\author{
ALPO KANKAANPERÄ, \\ TAUNO LAUNOSALO and \\ PENTTI SALOMAA
}

\author{
Department of Chemistry, University of \\ Turku, Turku, Finland
}

$T$ he first successful synthesis of 1,3dioxole (I) and one of its alkyl derivatives was reported a few years ago by Field. ${ }^{1}$ The reaction scheme consisted of a Diels-Alder addition of vinylene<smiles>C1OC2COC1O2</smiles>

carbonate to anthracene, hydrolysis of the adduct to a 1,2-diol, acetalization of the diol to give a 1,3-dioxolane derivative, followed then by thermal cracking of the latter to yield the 1,3-dioxole desired. Some 2,4-diaryl-substituted 1,3-dioxoles have been prepared recently by Ried and Omran ${ }^{2}$ from diazoketones and aromatic aldehydes.

For kinetic studies of the hydrolysis of 5-membered cyclic vinyl ethers an easy route to a number of alkyl-substituted 1,3-dioxoles was desired. In a recent paper ${ }^{3}$ it had been shown that the hydrolysis of vinyl ethers exhibits general acid catalysis involving a rate-determining proton transfer step which yields a mesomeric oxonium-carbonium ion as the first reaction intermediate. This reaction mechanism suggested that the intermediates generated by the hydrolysis of 4-alkyl-1,3-dioxoles (e.g., eqn. (II)) should be just the same as those formed from the respective 4alkylidene-1,3-dioxolanes (e.g., eqn. (III)). It was therefore inferred that, under conditions where the hydrolytic cleavage of the intermediates could not occur, 1,3-dioxoles and the alkylidene dioxolanes would be readily interconvertible by general acid catalysis. This was verified by the actual experiments. Because alkylidene dioxolanes can be easily obtained from 4- $\alpha$-chloroalkyldioxolanes, the con- 
<smiles>C1=C2COCO[C@H](C1)O2</smiles><smiles>C=C1COCO1</smiles>

version of the former to 1,3-dioxoles allowed a convenient preparation of these compounds. The method is naturally limited to such 1,3-dioxoles that are 4-alkyl-substituted.

The procedure chosen for the isomerization reaction was to boil under reflux one part of the alkylidene dioxolane with about 4 parts of ethylene glycol. The progress of the conversion was followed by gas chromatography and it was found that, depending on the alkylidene dioxolane used, 5 to $10 \mathrm{~h}$ were sufficient to complete its isomerization to the 1,3-dioxole. Practically no alkylidene dioxolane was left at the final equilibrium, which indicated that under these conditions 1,3-dioxoles are thermodynamically greatly favored over the corresponding alkylidene dioxolanes. Almost quantitative yields were hence obtained. No added strong acid was needed to induce the isomeriza. tion, the solvent itself being capable of acting as a general acid. On the contrary, it was found that under the influence of added strong acids, e.g., $p$-toluenesulfonic acid, substantial parts of the compounds polymerized by side reactions.

The details for two typical preparations of 1,3-dioxoles are given below. The kinetic and thermodynamic aspects of the reactions will be discussed in a subsequent paper.

4-Methyl-1,3-dioxole. 4-Methylene-1,3-dioxolane $(34 \mathrm{~g})$ was refluxed with ethylene glycol $(136 \mathrm{~g})$. The reaction was completed in $10 \mathrm{~h}$, after which the 1,3-dioxole formed was separated from the glycol by fractional distillation. The final purification took place over metallic sodium in a Todd precision fractionation assembly. The purity and identity of the purified 1,3-dioxole was independently verified by gas chromatography, infrared spectroscopy and kinetic experiments. B.p. $75.5-76.0^{\circ} \mathrm{C} 759$ torr, $n_{\mathrm{D}}{ }^{20} 1.3999$, $d_{4}^{20} 1.0646$.

2,4-Dimethyl-1,3-dioxole. The same procedures were followed starting from 2-methyl4-methylene-1,3-dioxolane (31 g) and ethylene glycol $(100 \mathrm{~g})$. In this case a period of $5 \mathrm{~h}$ was sufficient to complete the isomerization reaction. B.p. $82.0-82.5^{\circ} \mathrm{C} / 758$ torr, $n_{\mathrm{D}}{ }^{20}$ $1.3979, d_{4}{ }^{20} 0.9803$.

4-Methylene-1,3-dioxolanes. 4-Chloromethyl1,3-dioxolane and 2-methyl-4-chloromethyl1,3-dioxolane were prepared from 3-chloro1,2-propanediol and paraformaldehyde or paraldehyde, respectively. Equimolar mixtures of the reactants were refluxed with hexane and $p$-toluenesulfonic acid in an azeotropic water removal assembly until the completion of the acetal formation, after which the chlorodioxolanes were separated and purified by repeated distillations. To obtain the 4-methylene-1,3-dioxolanes desired, hydrogen chloride was eliminated from the chlorodioxolanes in a similar way as described by Hermann, Fischer and Feldmann." The following physical constants were recorded for the purified compounds: 4-methylene-1,3-dioxolane, b.p. $24-26^{\circ} \mathrm{C} / 45-50$ torr, $n_{\mathrm{D}}{ }^{20} 1.4348$, $d_{4}{ }^{20} \quad$ 1.0447; 2-methyl-4-methylene-1,3-dioxolane, b.p. $92-96^{\circ} \mathrm{C} / 760$ torr, $n_{\mathrm{D}}{ }^{20} 1.4230$, $d_{4}{ }^{20} 0.9805$.

Acknowledgement. The authors wish to thank the National Research Council for Sciences for financial support.

1. Field, N. D. J. Am. Chem. Soc. 83 (1961) 3504.

2. Ried, W. and Omran, J. Ann. 673 (1964) 120.

3. Salomaa, P., Kankaanperä, A. and Lajunen, M. Acta Chem. Scand. 20 (1966) 1790.

4. Hermann, O. L., Fischer, E. B. and Feldmann, L. Ber. B 63 (1930) 1732.

Received October 24, 1966.

Acta Chem. Scand. 20 (1966) No. 9 